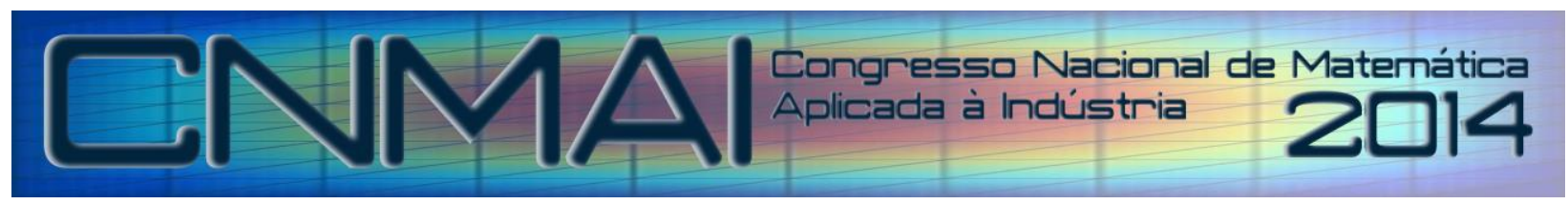

18 a 21 de novembro de 2014, Caldas Novas - Goiás

\title{
ACIONAMENTO DE GARRA ELETROMECÂNICA PELA INTERNET UTILIZANDO A PLATAFORMA RASPBERRY PI
}

\author{
Filipe Cavalcanti Fernandes, 13filipe11@ gmail.com ${ }^{1}$ \\ Alberto Chéquer Novaes Neto, alberto.chequer@gmail.com² \\ Thayanne Barros Bandeira, thayane.bandeira@hotmail.com ${ }^{3}$ \\ Wellington Fonseca, Fonseca@ufpa.br ${ }^{4}$
}

\begin{abstract}
${ }^{1}$ Universidade Federal do Pará - Campus Universitário de Tucuruí - Faculdade de Engenharia Elétrica - Rodovia BR
22 Km 13 - Canteiro de Obras - UHE Tucuruí - Vila Permanente 68464-000 - Tucuruí - Pará

${ }^{2}$ Universidade Federal do Pará - Campus Universitário de Tucuruí - Faculdade de Engenharia da Computação -

Rodovia BR 22 Km 13 - Canteiro de Obras - UHE Tucuruí - Vila Permanente 68464-000 - Tucuruí - Pará

${ }^{3}$ Universidade Federal do Pará - Campus Universitário de Tucuruí - Faculdade de Engenharia Elétrica - Rodovia BR

22 Km 13 - Canteiro de Obras - UHE Tucuruí - Vila Permanente 68464-000 - Tucuruí - Pará

${ }^{4}$ Universidade Federal do Pará - Campus Universitário de Tucuruí - Faculdade de Engenharia Elétrica - Rodovia BR 22 Km 13 - Canteiro de Obras - UHE Tucuruí - Vila Permanente 68464-000 - Tucuruí - Pará
\end{abstract}

Resumo: Esta pesquisa tem como objetivo demonstrar o controle de um braço robótico por meio de uma plataforma de prototipagem raspberry $\mathrm{P} i$, em comunicação com a internet, demonstrando uma solução simples e de baixo custo para mecatrônica aplicada a industria. Através de um web service administrado pelo Raspberry Pi, podemos comunicar o braço robótico de qualquer lugar de uma indústria, utilizando apenas um dispositivo que possua uma conexão wi-fi e esteja conectado em rede local. A garra proposta neste artigo serve apenas como exemplo, entretanto, qualquer outro dispositivo atuador pode ser acoplado, facilitando assim a adaptação deste projeto para várias situações reais.

\section{Palavras-chave: Raspberry Pi, Servidor Web,Garra eletro mecânica.}

\section{INTRODUÇÃO}

A tecnologia tem evoluído de tal forma, que tem afetado todas as áreas do conhecimento e do convívio humano. Segundo o dicionário Michaelis (Weiszflog) a tecnologia é o estudo sistemático dos procedimentos e equipamentos técnicos necessários para a transformação de matérias-primas em produtos. Por conseguinte, a tecnologia da Informação (TI) é a área do conhecimento responsável por criar, administrar e manter a gestão da informação através de dispositivos e equipamentos para acesso, operação e armazenamento dos dados, além de criar formas de gerir informação para tomada de decisão.

$\mathrm{O}$ avanço da tecnologia de informação acarretou na popularização e no barateamento das tecnologias. Por intermédio, criou um link entre equipamentos complexos e sociedade, além de aumentar o fluxo de informações e a disponibilização de equipamentos de forma fácil e acessível, tanto para a comunidade acadêmica de conhecimento elevado quanto para a comunidade em geral. Devido a evolução desta tecnologia, trabalhar e utilizar microcontroladores e microprocessadores tornou-se mais simples e perfeitamente eficaz, por exemplo.

Advindo dos benefícios, indústrias buscam continuamente por alternativas aprimoradas e modernas que possam baratear seus produtos de forma segura e sem perder a qualidade. É ampla a procura por modernização de procedimentos, serviços e equipamentos industriais que permitam solucionar estas questões, no entanto mantendo a segurança dos funcionários e prestadores de serviço.

Desta forma, faz-se necessário ações que evoluam progressivamente de modo a constituir uma série de outras ações para serem controladas a fim de alcançar um determinado resultado ou atingir uma meta (OGATA, 2000). Para efetuar estas ações é preciso controlar os processos atuando sobre eles, utilizando de todos os mecanismos disponíveis ou condições a que o mesmo está sujeito e deste modo alcançar os devidos fins como: a diminuição do custo de mão-deobra final do produto, a diminuição e prevenção de acidentes em funcionários humanos e produção em grande escala em tempo reduzido. 
Em consequência ao aumento da procura por estas tecnologias de automação industrial e robótica, diversas entidades públicas e privadas como: universidades, agências governamentais e empresas, incentivam a pesquisa, o desenvolvimento, e a criação de aplicações nessas áreas.

Portanto, vislumbrando a abordagem acima, a presente pesquisa traz o controle de um braço robótico por meio de uma plataforma de prototipagem Raspberry Pi, em comunicação com a internet. O projeto demonstra uma solução simples e econômica da mecatrônica aplicada para a indústria. O sistema dispõe de um serviço web administrado pelo Raspberry Pi que envia comandos ao braço robótico por todo o ambiente que tenha acesso a rede local da indústria, utilizando apenas um dispositivo que possua uma conexão wi-fi e esteja conectado em rede local. A garra proposta neste artigo serve apenas como exemplo, entretanto, qualquer outro dispositivo atuador pode ser acoplado ao sistema, facilitando assim a adaptação deste projeto para várias situações reais.

\section{METODOLOGIA}

O projeto teve seu desenvolvimento baseado em três sub etapas, implementadas gradualmente para melhor gerência do projeto. Para utilização da garra eletromecânica fez-se necessário o estudo de elementos elétricos e computacionais afim de consolidar um projeto efetivamente seguro e eficaz através da internet.

As etapas do desenvolvimento do protótipo fundamenta-se na criação de um sistema modelo para o controle de dispositivos via internet em rede local, o presente sistema adequa-se a qualquer tipo de dispositivo. Para a efetivação do método fez-se indispensável a utilização dos elementos: plataforma open source Raspberry Pi, Internet e os Atuadores, a garra mecânica, por exemplo. Para correta utilização dos elementos acima citados é importante detalhar algumas explicações conforme abaixo.

\subsection{RASPBERRY PI}

O Raspberry Pi conforme a figura 1, é um microcomputador de reduzidas dimensões, desenvolvido pela Raspberry Pi Foundation em conjunto a Universidade de Cambridge em 2012. Criado com o intuito de promover o estudo da ciência da computação nas escolas do ensino básico ao ensino secundário (Upton et al.,2014).

O hardware deste microcomputador é integrado numa única placa de circuito impresso e existem dois modelos: o A e o B, ambos usam processador multimídia Broadcom BCM2835 system-on-chip (SoC) de 700 Mhz com placa gráfica integrada VideoCore IV. Os modelos possuem uma entrada de cartão $S D$, devido não apresentar memória interna, portanto faz-se necessário adquirir para o armazenamento de dados, bem como a instalação do sistema operacional e aplicações. Os dispositivos A e B respectivamente possuem 256 e 512 MB de memória RAM, saídas de áudio, vídeo, rede, USB e mini USB, além de 26 pinos que sevem como entrada e saída, interligando diversos tipos de hardwares podendo controlá-los (Richardson et al.,2013).

O Raspberry Pi possui um processador ARM, logo, não é possível instalar o sistema operacional Windows. No entanto, estão disponíveis gratuitamente sistemas operacionais baseados no Linux compatíveis com este microcomputador. O Raspbian representado na figura 2, é um exemplo de sistema operacional, uma distribuição Linux baseado em Debian que no site do Raspberry é distribuído o New Out Of Box Software (NOOBS), com boa parte dos programas pré-instalados facilitando a utilização pelo usuário (Horan et al.,2013).

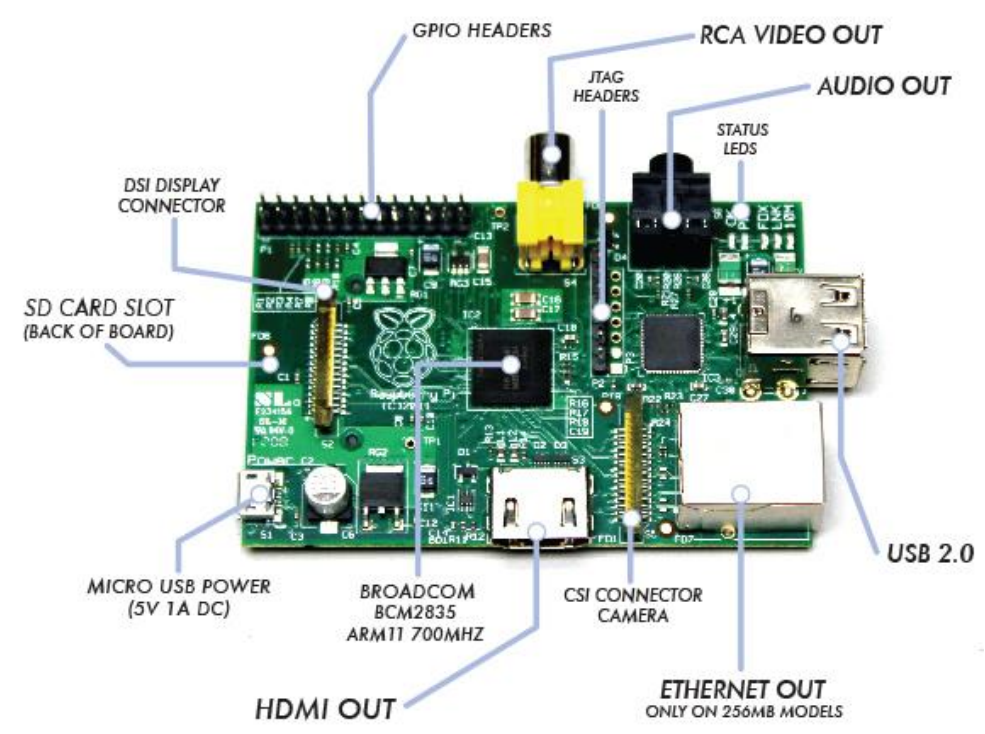

Figura 1: Raspberry Pi modelo B 


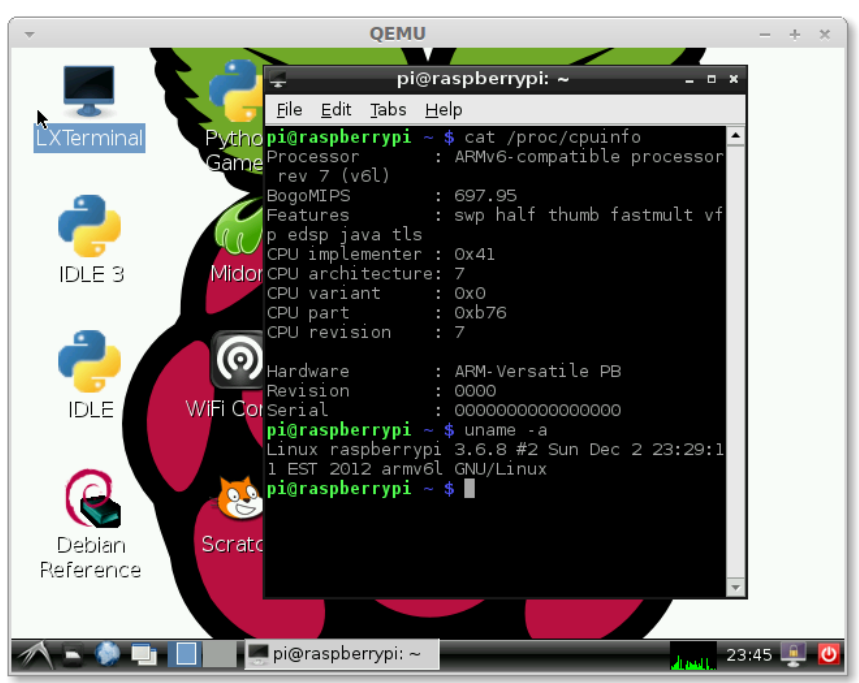

Figura 2: Área de Trabalho do Raspbian com Terminal em execução

\subsection{INTERNET}

Segundo Paulo J.(2011), as tecnologias de informação e de comunicação abrangem novas perspectivas à sociedade do futuro. A informação uma vez produzida, circula instantaneamente para receptores, que tratam e incorporam em esquemas lógicos e científicos. Na sequência é transformada por cada usuário em conhecimento. Dentre as possibilidades presentes na rede, conectar o Raspberry é uma das mais fascinantes, pois amplia a potencialidade de aplicações existentes. No entanto antes de integrá-lo a rede mundial de computadores é necessário conectá-lo à nossa rede local, tal façanha pode ser atingida utilizando a porta ethernet, necessitando apenas de um cabo de rede RJ-45, padrão para redes ethernet, e conectá-lo ao Raspberry.

Um grupo de computadores conectados entre si e que podem comunicar-se um com o outro e compartilhar recursos como impressoras em uma área local, denomina-se uma rede local ou $L A N$. Os dados são enviados na forma de pacotes e, para controlar a transmissão dos pacotes, podem ser usadas diferentes tecnologias. A tecnologia $L A N$ mais utilizada é a Ethernet, especificada em um padrão chamado IEEE 802.3. Para configurar nossa $L A N$ é necessário um roteador, afim de que ele crie uma rota para nosso Raspberry quando quisermos conectá-lo a Internet, para então prover o servidor Web que trabalha com os protocolos TCP e UDP (Axis, 2013).

O Protocolo de Controle de Transmissão (TCP) e o Protocolo de Datagramas de Usuário (UDP) são os IPs (Protocolos de Internet) usados para enviar dados. Esses protocolos atuam como transportadores para muitos outros protocolos. Por exemplo, o HTTP (Protocolo de Transferência de Hipertexto), usado para navegar por páginas da Web em servidores de todo o mundo através da Internet, é transportado pelo TCP (Axis, 2013). Já os servidores Web são responsáveis por armazenar e trocar informações com outras máquinas. Desta forma, pelo menos um cliente e um servidor são envolvidos em cada troca de informação: um cliente, que solicita informações, e um servidor que atende a esses pedidos.

Portanto, o Raspberry funcionará como um servidor, as páginas HTML (Linguagem de Marcação de Hipertexto) estarão hospedadas. Desta maneira, qualquer computador conectado à rede poderá solicitar e controlar o atuador que estará conectado com o Raspberry. É importante salientar que o Raspberry preliminarmente vincula-se a rede intranet, rede de computadores privada, que abrange as mesmas funcionalidades da internet com acessos restritos.

\subsection{ATUADOR}

Atuadores são dispositivos que transformam uma forma de energia em outra. Eles são utilizados na robótica para entregar à planta a excitação necessária para seu funcionamento, na forma do tipo de energia adequado. Por exemplo, se o funcionamento da planta estiver baseado em algum movimento dela ou de alguma das suas partes, serão necessários atuadores que forneçam energia mecânica para gerar esse movimento. Se a planta for um sistema térmico, será necessário um atuador que forneça a energia térmica necessária para ela atingir a temperatura desejada como resposta.

Os atuadores são divididos em três categorias: hidráulicos, pneumáticos e elétricos. O presente projeto utilizará um atuador elétrico, que funciona empregando energia elétrica. A princípio o projeto consisti no acionamento de uma garra mecânica através da página web armazenada no Raspberry. O acionamento ocorrerá por qualquer computador conectado à rede. A garra é feita de material reciclado proporcionando assim menor custo, além de ser ecologicamente correta.

A garra eletromecânica possui uma pinça, dois motores e pode fazer até quatro tipos de movimentos: Cima, baixo, abre e fecha a pinça. Conforme representada na figura 3 (a) (b) (c) (d): 


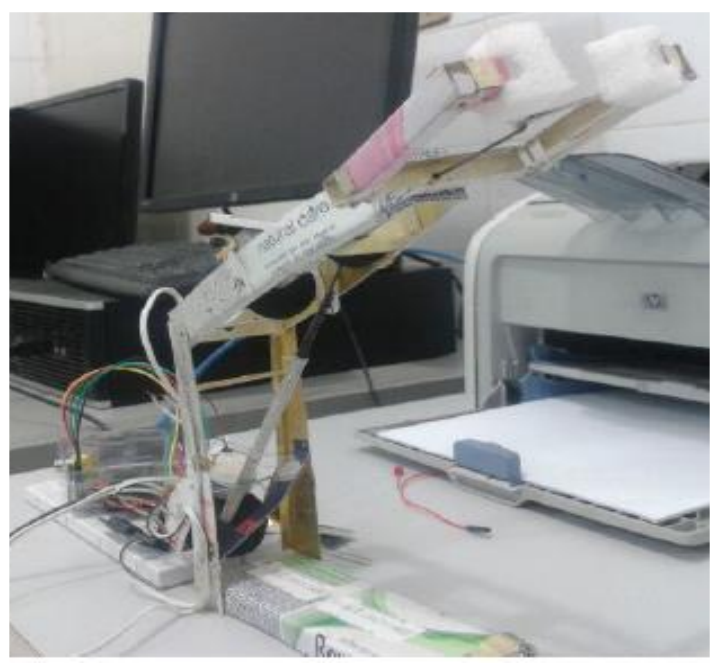

(a) Representa o braço da garra de movimentando para cima.

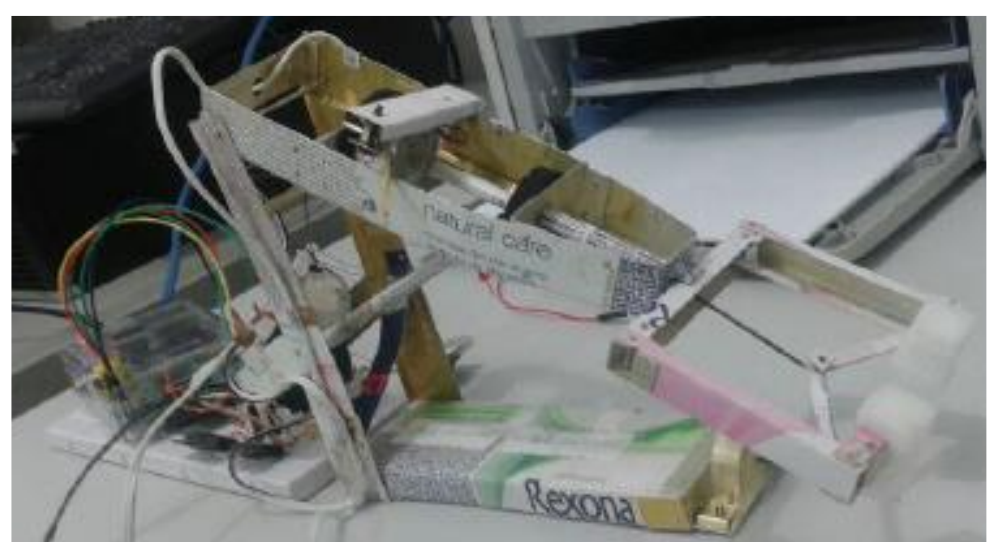

(b) Representa o braço da garra se movimentando para baixo.

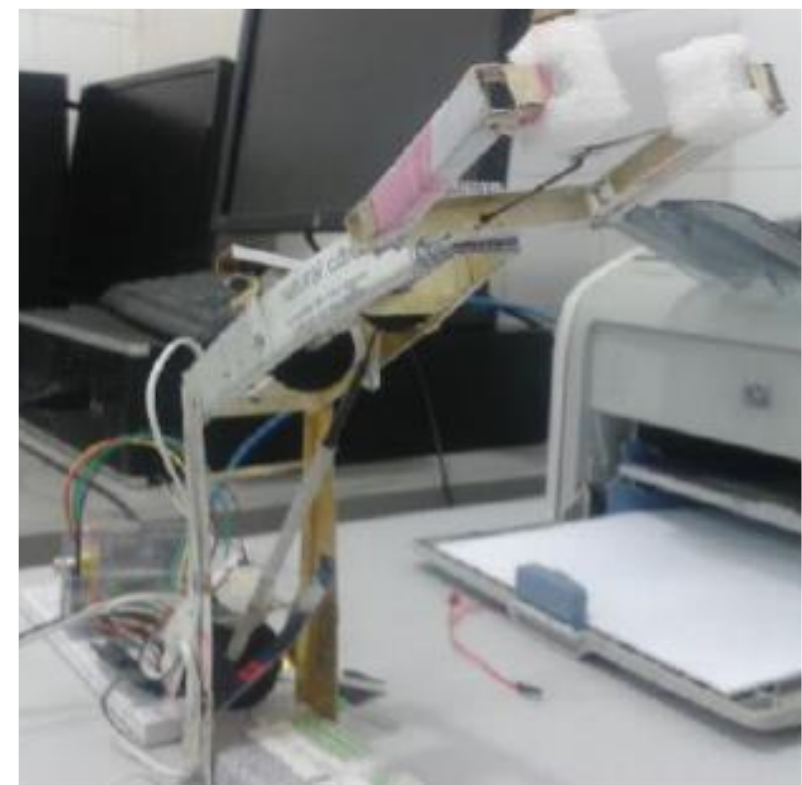

(c) Representa a pinça da garra se abrindo. 


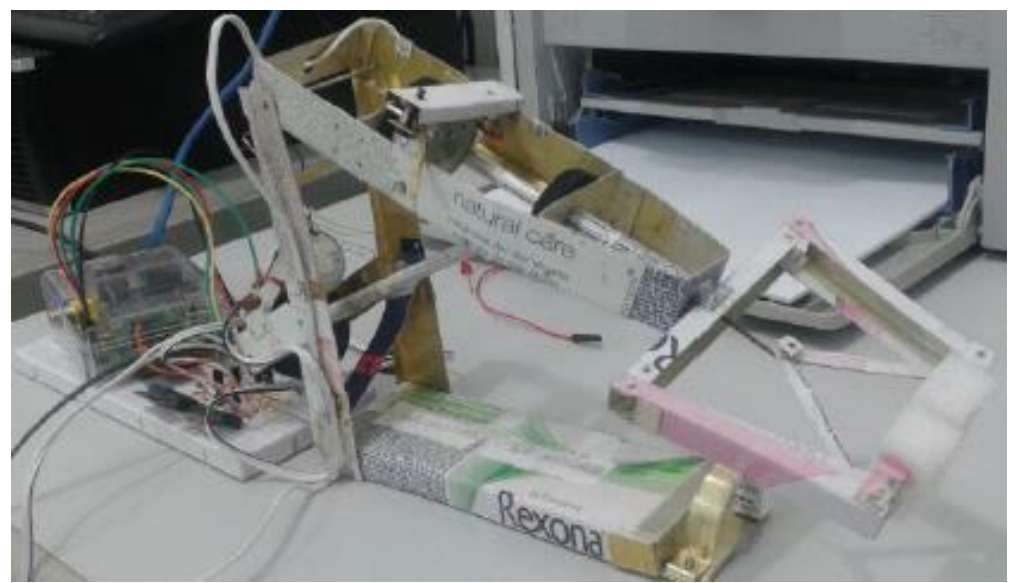

(d) Representa a pinça da garra se fechando.

Figura 3: Representa os movimentos do atuador escolhido, a Garra Eletromecânica.

\section{MONTAGEM}

Para realizar a montagem e configuração do sistema de forma adequada é importante organizar os equipamentos. O protótipo final simula o controle de uma garra eletromecânica através da rede local. É possível controlar o atuador por uma página web desenvolvida utilizando HTML (Linguagem de Marcação de Hipertexto) e CSS (linguagem de folhas de estilo). Para o devido funcionamento do protótipo foram utilizados os seguintes equipamentos listados abaixo:
- Cabos jumper
○ 1 Raspberry Pi
○ 1 Cartão sd
- 1 Garra Mecânica
○ 8 Transistores
○ 4 Resistências
○ 1 Protoboard
○ 1 Cabo RJ-45
○ 1 Roteador

\subsection{CIRCUITO}

$\mathrm{Na}$ montagem da central de gerenciamento do protótipo, o circuito é composto por 2 pontes $\mathrm{H}$ e 2 motores. A Ponte-H é um circuito simples, composta de 2 transistores NPN e 2 transistores PNP, capaz de controlar motores de corrente contínua diretamente utilizando sinais elétricos, permitindo o controle do sentido da rotação, da potência e velocidade.

O Raspberry é alimentado através de um cabo mini USB, e posteriormente precisa ser conectado ao roteador através de um cabo RJ-45, cada motor está conectado a uma ponte $\mathrm{H}$ utilizada no projeto para controlar o giro e a alimentação do motor, visto que o Raspberry não tem a tensão necessária.

$\mathrm{Na}$ figura 4 é possível visualizar o circuito elaborado com as conexão do Raspberry com o papel de servidor e as duas pontes $\mathrm{H}$. Na figura 5 está a representação esquemática do circuito. 


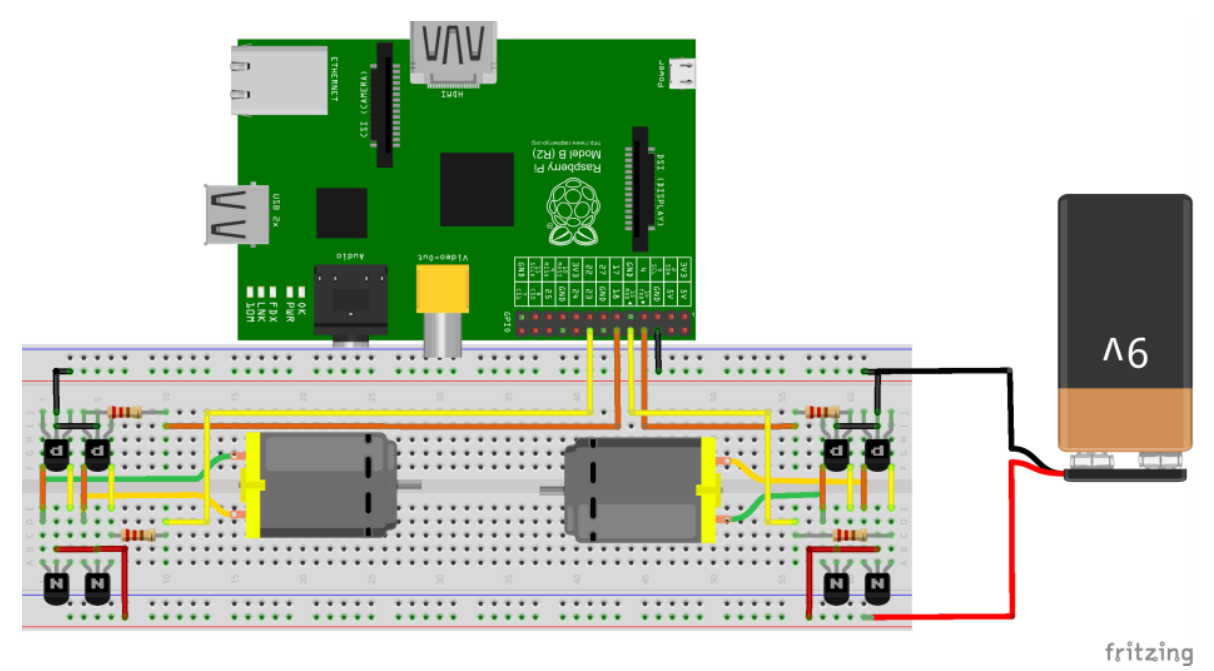

Figura 4: Representa o circuito elaborado.

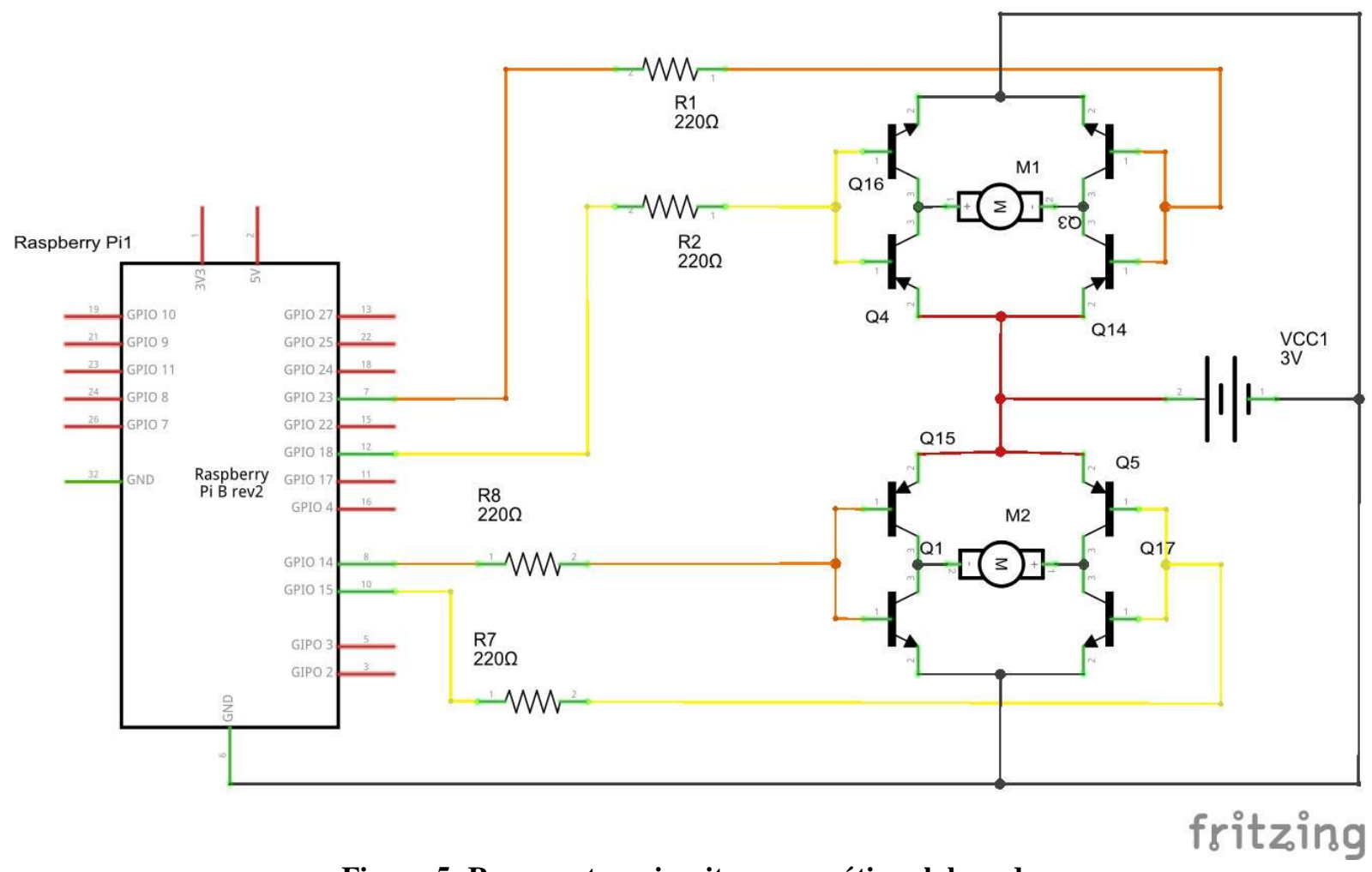

\subsection{PROTÓTIPO WEB}

O presente protótipo teve sua construção inteiramente na linguagem HTML com apoio de formatações em CSS. O HTML é uma linguagem de marcação de texto, em que são possíveis a criação de diversas formas de páginas web, utilizando tags, ou seja, rótulos usados para apresentar o website. A figura 6 mostra o layout da página principal com quatro botões e cada um referente a um comando de controle da garra eletromecânica, através dessa página o usuário pode acionar os comandos.

A figura 7 mostra o diagrama da rede local, com fluxo das informações entre nossos dispositivos, após conectar o Raspberry na rede através do cabo RJ-45 no roteador é necessário nas configurações criar arquivos em python que direcionam o IP para a rede local. Dessa forma qualquer dispositivo que for capaz de acessar a rede terá acesso à página hospedada no Raspberry que é o servidor, quando algum usuário clica no botão na página web, imediatamente uma solicitação é enviada ao Raspberry que recebe a informação e a processa gerando o comando para o atuador, acionando e posteriormente enviando de volta a resposta para o usuário. 


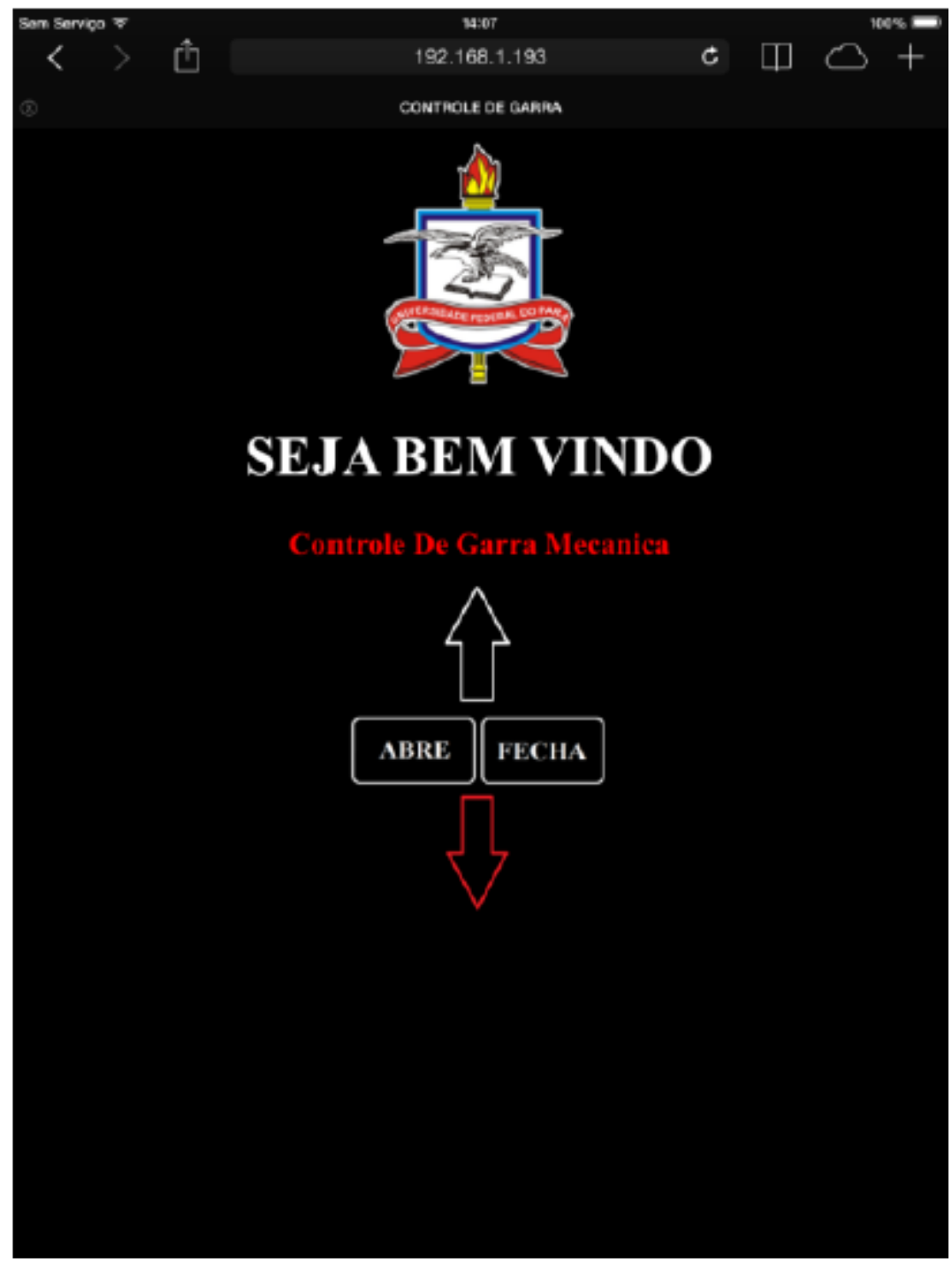

Figura 6: Página web principal

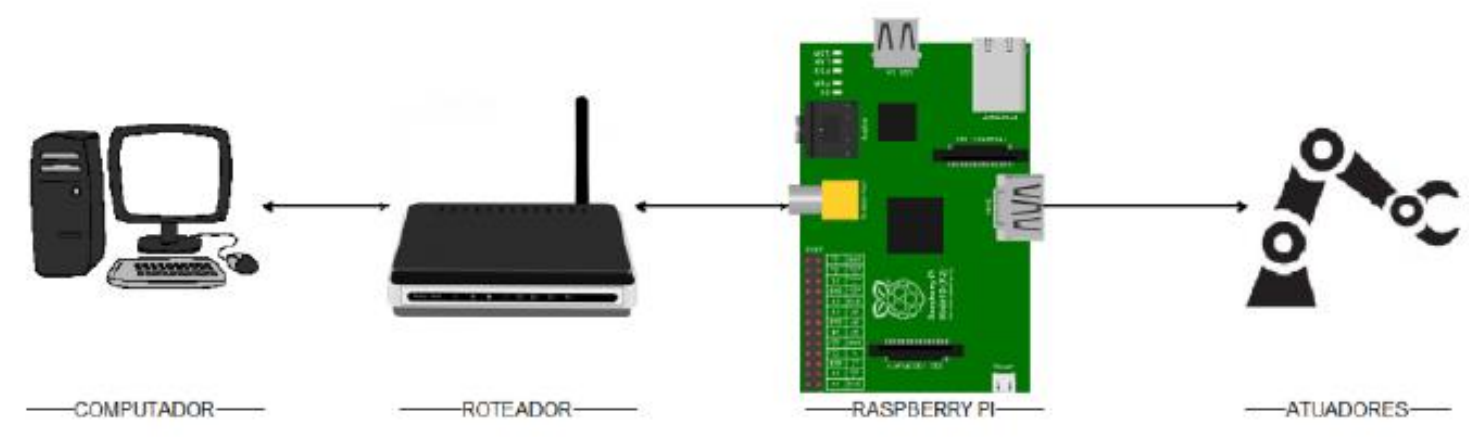

Figura 7: Diagrama de fluxo da rede local

\subsection{ALGORITMO IMPLEMENTADO}

A lógica computacional elaborada na figura 8 foi realizada através da combinação entre a linguagem de programação python e HTML no próprio Raspberry.

O Raspberry vai receber as requisições da internet, processar as informações, e acionar os atuadores através dos seguintes passos:

- O microcomputador inicia o programa, definimos as variáveis e pinos do Raspberry possibilitando o acionamento do servidor, onde o programa atribui ao Raspberry uma porta no roteador que pode ser configurada pelo programador, necessário para a comunicação com o roteador. 
- Com o servidor inicializado, a página é disponibilizada na rede, dessa forma o usuário poderá enviar as solicitações de controle para o Raspberry. Cada botão correspondente aos movimentos da garra possuem um código diferente. Quando o usuário clica em um botão, seu código é enviado ao raspberry que faz o processamento e aciona a respectiva funcionalidade.

- Após a solicitação ao servidor e processamento dos dados, uma resposta à solicitação é enviada, este procedimento não é mostrado ao usuário, apenas é mostrado ao programador que controla o servidor, depois de tais procedimentos o usúario pode verificar o acionamento do led.

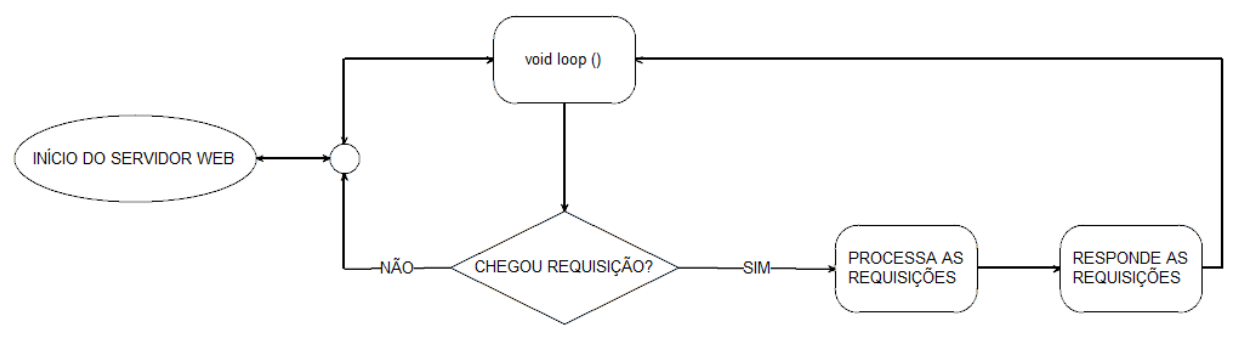

Figura 8: Representa Lógica computacional do Servidor

\section{RESULTADOS}

Foi elaborada satisfatoriamente o controle do braço robótico utilizando o Raspberry Pi como servidor conectado a uma rede intranet, possibilitando assim que qualquer pessoa com dispositivo com conexão wi-fi possa controlar a garra ou qual quer outro dispositivo atuador.

O alcance do controle é restringido ao sinal emitido por um roteador. No caso de uma fábrica de grandes proporções se faria necessário a implementação de um sistema de repetição de sinal para que abrangesse toda a fábrica de forma satisfatória.

Mesmo desenvolvendo o projeto de maneira satisfatória é importante ressaltar alguns aspectos que podem ser aperfeiçoados. Um exemplo claro disso é a não ligação do projeto com a rede mundial de computadores (internet), restringindo assim liberdade para um gerenciamento dos atuadores fora das dependências da fabrica. Porém a utilização apenas da intranet se deu por causa da impossibilidade de abertura de uma porta de comunicação, foram buscadas várias formas de conexões, sem sucesso decidimos fazer um estudo mais aprofundado nessa área para posteriores projetos.

Outro ponto de melhoria é a elaboração de um sistema de segurança que delimite o controle da garra ou atuadores para apenas as pessoas devidamente autorizadas, gerando segurança para os utilizadores do sistema. $\mathrm{O}$ que nos norteou na elaboração da primeira versão desta proposta foi a movimentação de um atuador da utilizando a intranet, ponto este que foi atingido, porém ficam alguns pontos a serem resolvidos posteriormente com a elaboração de uma segunda versão deste projeto.

Por fim, devemos ressaltar que a garra utilizada neste projeto tem apenas um papel demonstrativo, pois não possui robustez para a implementação em uma industria real. Mas apenas esse fato não é suficiente para anular os resultados obtidos com o sistema.

\section{COSIDERAÇÕES FINAIS}

A medida que a tecnologia de informação evolui, surgem distintas inovações em conseqüência ao aumento da procura por tecnologias de automação industrial e robótica. Esses conceitos evoluem e integram eletrônica e programação objetivando a melhoria dos processos industriais, buscando garantir condições essenciais como: agilidade, qualidade, redução de custo e a diminuição de acidentes nas áreas de atuação.

Desta forma ao fim do trabalho a garra eletromecânica servirá como plataforma de desenvolvimento para aplicações de manipuladores robóticos, bem como permitir a análises com uma visão computacional de controle de manipuladores à distância. Alcançando o principal objetivo de simular um sistema de controle robotizado num processo industrial fictício, através da internet, introduzindo uma forma de manipular sistemas eletromecânicos utilizando o Raspberry Pi.

Por fim, os objetivos propostos na especificação inicial foram satisfeitos e com o sucesso é possível expandir para outros dispositivos e setores.

\section{REFERENCIAS}


Axis Communications < http://www.axis.com/pt/products/video/about_networkvideo/internet.htm> Acesso em 03 jun: 2014

Uptor E. Hafacree G. 2014. Raspberry Pi Guia do Usuário. Reino Unido. 2014. v.2 n.1 p. 14-33.

Escola Superior de Tecnologia e Gestão de Viseu, Disponível em:

http://www.estgv.ipv.pt/paginaspessoais/quental/trabs/psi/internet.htm> Acesso em: 15 mai. 2014

Germany Trade \& Invest, Disponível em:

<http://www.gtai.de/GTAI/Navigation/DE/Trade/maerkte,did=910176.html> Acesso em: 10 jun: 2014.

Honran B, 2013. Raspberry Pi Prefácio. Estados Unidos. 2013. v.1 n.1 p.1-17.

Home Toys, Disponível em:

<http://www.hometoys.com/emagazine/2014/02/36-million-homes-in-europe-and-north-america-will-be-smart-by2017/2235> Acesso em: 10 jun: 2014

OGATA, K. Engenharia de Controle Moderno. 3. Ed. Rio de Janeiro: Livros Técnicos e Científicos, 2000. 813p.

Pazos, Fernando. Automação de sistemas \& robótica - Axel Books, 2002.

Richardson M., Shaum W.. Getting Started With Raspberry Pi. Estados Unidos. 2013. v.1 n.1 p.34-49

Weiszflog, Walter. Michaelis. São Paulo: Melhoramentos, 1998.

\title{
ACTIVATION OF ELETRICMECHANICAL GRAB THROUGH THE INTERNET USING THE PLATFORM RASPBERRY PI
}

\author{
Filipe Cavalcanti Fernandes, 13filipe11@gmail.com ${ }^{1}$ \\ Alberto Chéquer Novaes Neto, alberto.chequer@gmail.com² \\ Thayanne Barros Bandeira, thayane.bandeira@ hotmail.com $^{3}$ \\ Wellington Fonseca, Fonseca@ufpa.br ${ }^{4}$
}

\begin{abstract}
${ }^{1}$ Universidade Federal do Pará - Campus Universitário de Tucuruí - Faculdade de Engenharia Elétrica - Rodovia BR
22 Km 13 - Canteiro de Obras - UHE Tucuruí - Vila Permanente 68464-000 - Tucuruí - Pará

${ }^{2}$ Universidade Federal do Pará - Campus Universitário de Tucuruí - Faculdade de Engenharia da Computação -

Rodovia BR 22 Km 13 - Canteiro de Obras - UHE Tucuruí - Vila Permanente 68464-000 - Tucuruí - Pará

${ }^{3}$ Universidade Federal do Pará - Campus Universitário de Tucuruí - Faculdade de Engenharia Elétrica - Rodovia BR

22 Km 13 - Canteiro de Obras - UHE Tucuruí - Vila Permanente 68464-000 - Tucuruí - Pará

${ }^{4}$ Universidade Federal do Pará - Campus Universitário de Tucuruí - Faculdade de Engenharia Elétrica - Rodovia BR 22 Km 13 - Canteiro de Obras - UHE Tucuruí - Vila Permanente 68464-000 - Tucuruí - Pará
\end{abstract}

\begin{abstract}
This research aims to demonstrate the control of a robotic arm through a prototyping platform Raspberry Pi, in communication with the internet, demonstrating a simple and inexpensive solution for mechatronics applied to industry. Through a web service administered by the Raspberry Pi, the robotic arm can communicate from anywhere in an industry, using only one device that has a wi-fi connection and is connected in LAN. The Grab proposed in this paper serves only as an example, but any other device may be coupled actuator, thereby facilitating the adaptation of this project for several real situations.
\end{abstract}

Keywords: Raspberry Pi, Web Server, electromechanical grab. 